\title{
UNE PARODIE DE GENRE ? L'ÉPISTOLARITÉ DES LETTRES D'AMABED, ETC. (1769) ENTRE MONTESQUIEU, GRAFFIGNY ET RICHARDSON
}

\author{
Laurence DAUBERCIES \\ Université de Liège
}

\begin{abstract}
En): Les Lettres d'Amabed, etc. is an epistolary tale written by Voltaire and first published in 1769. While its narrative appears to be a partial rewriting of Madame de Graffigny's Lettres d'une Péruvienne, several aspects of its general structure reflect well-known archetypes of the epistolary genre. More than a canonic attempt at creating an epistolary novel, the tale appears rather as a badinage with the traditional codes of epistolary writing. It particularly resonates with two novels that embody the dominant paradigms of the epistolary field: the "exotic" vein characterized by the Lettres Persanes (Montesquieu, 1721) and the "sentimental" one with Pamela (Richardson, 1740). This study seeks to demonstrate how the in praesentia use of epistolary codes creates a parodic filiation with the former, whereas the in absentia game with epistolary voices implicitly satirizes the latter. With this case study, we ultimately hope to outline how the moral and critical realism associated with the epistolary genre - and its subgenres - can transcend its narrative content to affect its very own structures and writing codes, from a parodic and metacritical point of view.
\end{abstract}

Keywords (En): philosophical tale; epistolary novel; parody; literary genres; Les Lettres d'Amabed; Voltaire; Montesquieu; Richardson; Graffigny.

Mots-clés (Fr) : conte philosophique ; roman épistolaire ; parodie ; genres littéraires ; Les Lettres d'Amabed ; Voltaire ; Montesquieu ; Richardson ; Graffigny.

\section{Une forme inédite : le conte épistolaire}

Les Lettres d'Amabed, etc., conte voltairien publié pour la première fois en 1769 , se présente sous la forme d'un échange épistolaire entre, d'un côté, Amabed et Adaté, un jeune couple d'Orientaux voyageant vers l'Europe, et, de l'autre, leur père spirituel Shastasid, resté au pays. Les jeunes gens relatent avec candeur leurs impressions de voyage et les (més)aventures vécues en route vers l'Occident. Pardelà le récit naïf des deux protagonistes se dessine une satire de la société européenne et de l'hypocrisie catholique, selon le modèle traditionnel du conte philosophique voltairien. Initialement purs et vertueux, les épistoliers subiront ainsi d'injustes persécutions - emprisonnements, viols, calomnies et manipulations avant de se laisser gagner par les charmes vénéneux de l'Occident, faits de carnivorisme, de frivolité, d'hypocrisie et de luxure.

Ce texte facétieux écrit par un Voltaire vieillissant a été peu apprécié par la critique de son temps. Diderot a ainsi déclaré qu'il n'avait «nul intérêt » (MERVAUd, 1991 : 76). Peu d'ailleurs l'ont commenté au cours des dernières décennies (HOWELLS, 1987 ; MALLINSON, 2008 ; TOUMARKINE, 1998). Il interpelle cependant par sa structure unique, puisqu'il s'agit du seul conte voltairien adoptant une structure épistolaire ${ }^{1}$. Comme nous allons le voir, le conte constitue en réalité, par de nombreux aspects, un prisme humoristique réfractant plusieurs codes d'écritures du roman par lettres. Son traitement spécifique de l'épistolarité fait ainsi

\footnotetext{
${ }^{1}$ La Lettre d'un Turc (1750), autre conte voltairien, ne peut être considérée comme un roman épistolaire, puisqu'elle se compose d'une unique missive et ne met pas en scène de véritable échange. Echo des études romanes XII/1-2, 2016 
écho aux caractéristiques de plusieurs représentants archétypaux du genre, qui occupait alors les devants de la scène littéraire. Ainsi, l'exploitation en présence de certains codes rédactionnels joue ouvertement avec les poncifs de la veine épistolaire exotique (popularisée avec la parution des Lettres Persanes de Montesquieu en 1721), tandis que l'absence et la disparition d'autres composantes constituent un écho parodique à certains poncifs d'écriture de la veine épistolaire sentimentale $^{2}$ (venue d'Angleterre via la traduction de Paméla ou la vertu récompensée de Samuel Richardson, en 1741). Il s'agira ainsi de mettre au jour les modalités d'une exploitation parodique et métacritique des codes d'écriture réalistes inhérents au genre - et aux sous-genres - épistolaires.

\section{L'épistolaire in praesentia : codes et réécritures du genre «persan » ou « exotique »}

\subsection{Les Lettres d'Amabed et la veine exotique}

À la fin du XVII ${ }^{\mathrm{e}}$ siècle, les exigences de vérité, d'honnêteté et d'authenticité prônées par la doctrine littéraire classique entraînent la dissimulation progressive de la fiction romanesque derrière le masque réaliste de la forme épistolaire. Offrant un accès direct à l'intériorité de l'épistolier et dispersant la fonction narrative entre divers personnages, la missive dérobe auteur et narrateur à la perception immédiate du lecteur, créant «l'illusion de l'authenticité » (VeRsinI, 1979). L'une des premières occurrences de ces nouvelles fictions adoptant la forme épistolaire est les Lettres portugaises, publiées anonymement en 1669, et qui passeront longtemps pour authentiques. La popularité grandissante de cette forme n'est pas sans nourrir de lien avec les grandes découvertes et le repoussement des limites géographiques propres à cette époque : l'Homme est poussé à témoigner de son expérience de la diversité humaine dans des lettres de voyages, qui se multiplient de manière exponentielle (GRASSI, 1998 : 112). La fiction ne tarde pas à les imiter. C'est ainsi que paraissent, en 1721, les Lettres Persanes de Montesquieu. Ce roman présente les lettres écrites par deux Perses, Usbek et Rica, qui voyagent en Europe et décrivent à leurs proches restés aux pays leur perception - à la fois candide et critique - des us et coutumes de nos régions. La critique sociale se déguise ici en correspondance. Le roman de Montesquieu fera de nombreux émules relevant, à l'instar des Les Lettres d'Amabed, du sous-genre dit «persan » ou « exotique »' tel que le décrit Frédéric Calas dans son étude typologique des romans épistolaires (CALAS, 1996 : 76-85).

Il apparaît que Les Lettres d'Amabed parodie la veine persane à plusieurs niveaux explicites. Au niveau thématique et narratif tout d'abord, par la réécriture partielle des Lettres d'une Péruvienne de Françoise de Graffigny. Au niveau structurel ensuite, par l'exécution caricaturale de plusieurs codes d'écriture identifiables remontant au modèle de Montesquieu.

\footnotetext{
${ }^{2}$ L'un des grands représentants français du genre sera Jean-Jacques Rousseau avec La Nouvelle Héloïse (1761).
} 


\subsection{Réécriture des Lettres d'une Péruvienne}

Jonathan Mallison, éditeur moderne des Lettres d'une Péruvienne (GRAFFIGNY, 2002), a mis en évidence l'existence de frappantes similitudes entre la trame narrative du conte voltairien et celle du roman épistolaire de son amie Françoise de Graffigny, publié en 1747 (MALLINSON, 2008). Ce dernier appartient lui aussi à la veine exotique, et la filiation générique entre les deux œuvres est communément acceptée (DOBIE, 1996 ; VolPILHAC-AUGER, 2004). Le texte de Graffigny, qui connut un énorme succès de librairie (GRAYSON, 1996 : 87-93 ; SHOWALTER, 2004 : 151-158 ; SMITH, 1990), met en scène Zilia, une jeune Indienne capturée par les Espagnols lors de la conquête du Pérou. À la suite de cet enlèvement, la jeune fille commence à raconter à son bien-aimé Aza, resté au pays, les tourments endurés durant son voyage. En chemin vers l'Europe, Déterville, capitaine d'un navire français, s'éprend d'elle, la ramène en France et la présente à sa famille. Fidèle à son premier amour et désireuse de retrouver sa patrie, Zilia lui résiste. Elle découvre - et relate - toutefois la vie française. Après avoir eu vent de l'infidélité de son fiancé Aza, la jeune femme décide de rester en France. Elle renonce cependant au mariage et choisit de mener une vie cultivée et indépendante, tout en priant Déterville de lui conserver son amitié. Jonathan Mallison a bien montré comment Voltaire réécrit ou adapte avec humour plusieurs composantes narratives du roman à succès : son héroïne, comme celle de Graffigny, est séparée de son bien-aimé ; toutes deux voguent vers l'Europe sur des bateaux qui seront attaqués ; toutes deux sont vulnérables face au désir de leurs «protecteurs » européens (Déterville pour Zilia et le père Fa tutto pour Adaté); et toutes deux insistent à plusieurs reprises sur les émotions vives associées à l'acte d'écriture épistolaire. Par ailleurs, les deux récits se clôturent par le passage des héroïnes dans une maison de campagne. Pour l'épistolière de Graffigny, la campagne est associée à une révélation identitaire ainsi qu'à un accès à l'indépendance financière et morale. Pour celle de Voltaire, le séjour à la campagne en compagnie de l'époux retrouvé et de deux prélats catholiques vire à la partie à quatre, épitome de la corruption morale. Cette distorsion parodique finale est représentative du ton général de la réappropriation voltairienne, dans laquelle " des épisodes dramatiques du texte de Graffigny sont retravaillés et trivialisés » (MALLINSON, $2008: 228$, nous traduisons).

\subsection{Caricature du modèle générique de Montesquieu}

Outre cette déformation ludique du schéma narratif spécifique des Lettres d'une Péruvienne, le conte de Voltaire parodie plus largement certaines composantes génériques archétypales du type exotique. Plusieurs poncifs du genre font ainsi l'objet d'un traitement ostensiblement caricatural, tels que la description des perceptions des voyageurs étrangers, la présentation du système de datation exotique, ou encore la mise en scène de l'éditeur fictif en traducteur et commentateur du texte. Afin de mettre ces détournements en évidence, il convient d'analyser Les Lettres d'Amabed en vis-à-vis du modèle prototypique que constituent les Lettres Persanes. Notons que le texte de Montesquieu est ici envisagé comme contrepoint représentatif des caractéristiques constitutives du paradigme 
générique détourné, et non en tant que source directe d'intertextualité avec le conte. Ce parallèle est des plus pertinent dès lors que l'on connaît l'attitude défiante souvent affichée par Voltaire envers l'œuvre de Montesquieu. Catherine Volpilhac-Auger signale ainsi que la correspondance de Françoise de Graffigny pour l'hiver 1738-1739 (époque à laquelle elle résidait à Cirey avec Voltaire et Madame du Châtelet) relate l'existence de débats animés entre l'épistolière et son illustre hôte, qui semble avoir qualifié de « fretin » le célèbre roman de Montesquieu (VOLPIHAC-AUGER, $2004: 160$ ). Voltaire l'égratignera à nouveau dans une lettre de 1743, soulignant « la frivole imagination » (D2748) à l'œuvre dans les Lettres persanes. La filiation générique avec Montesquieu était en outre évidente pour les lecteurs lors de la parution des Lettres d'Amabed, comme en témoigne ce commentaire - peu flatteur - extrait de la correspondance de Diderot :

Je sais bien que je vais faire jeter les hauts cris à tous les adorateurs de Voltaire, mais je vous dirai à l'oreille que ce nouvel ouvrage est sans goût, sans finesse, un rabâchage de toutes les vieilles polissonneries que l'auteur a débitées contre Moïse et Jésus-Christ, les prophètes et les apôtres, l'Église, les papes, les cardinaux, les prêtres et les moines ; nul intérêt, nulle chaleur, nulle vraisemblance, force ordures, une grosse gaîté. Il ne faut comparer cela ni à Zadig, ni à Memnon, ni à L'ingénu. C'est pourtant Voltaire, mais c'est la meilleure réparation qu'il pût faire à l'auteur des Lettres persanes. Si l'on y reconnaît par-ci par-là l'ongle du lion, c'est l'ongle du lion caduc (cité dans MERVAud, 1991 : 76).

L'une des caractéristiques principales du sous-genre persan est la manière dont il exploite la figure de la naïveté et de la présupposée inadéquation du voyageur étranger pour mettre en question certains aspects de la culture occidentale (CALAS, 1996 : 80). Ainsi, «dans le genre de la lettre écrite depuis l'étranger, ces sujets [politique, religion, philosophie] sont tous observés avec un œil très critique, bien que supposément naïf. L'étranger qui écrit au pays mesure en effet l'ancien système qui lui est familier par rapport au nouveau au sein duquel il est un étranger et, ce faisant, établit les mérites relatifs de chacun et tente de concilier leurs différences » (Howland, 1991 : 54, je traduis). Dans Les Lettres d'Amabed, Voltaire pousse le procédé à la limite de la vraisemblance, comme l'illustre la comparaison de ces extraits traitant de la richesse de l'Église catholique.

Voltaire, Les Lettres d'Amabed, etc.

Je lui disais : «Votre Dieu est né dans une étable entre un bœuf et un âne, il a été élevé, a vécu, est mort dans la pauvreté ; il a ordonné expressément la pauvreté à ses disciples ; il leur a déclaré qu'il n'y aurait parmi eux ni premier, ni dernier, et que celui qui voudrait commander aux autres les servirait [...]

- Tout cela est vrai, m'a-t-il répondu. Notre Dieu n'a pas commandé à nos maîtres formellement de s'enrichir aux dépens des peuples, et de ravir le bien d'autrui ; mais il l'a commandé virtuellement. Il est né entre un bœuf et un âne ; mais trois rois sont venus l'adorer dans une écurie. Les bœufs et les ânes figurent les peuples
Montesquieu, Lettres Persanes

Ce n'est pas tout. Les dervis ont en leurs mains presque toutes les richesses de l'État; c'est une société de gens avares, qui prennent toujours, et ne rendent jamais ; ils accumulent sans cesse des revenus, pour acquérir des capitaux. Tant de richesses tombent, pour ainsi dire, en paralysie ; plus de circulation, plus de commerce, plus d'arts, plus de manufactures (260). 
que nous enseignons ; et les trois rois figurent tous les monarques qui sont à nos pieds. Ses disciples étaient dans l'indigence ; donc nos maîtres doivent aujourd'hui regorger de richesses. Car, si ces premiers vice-Dieu n'eurent besoin que d'un écu, ceux d'aujourd'hui ont un besoin pressant de dix millions d'écus. Or, être pauvre, c'est n'avoir précisément que le nécessaire. Donc nos maîtres, n'ayant pas même le nécessaire, accomplissent la loi de la pauvreté à la rigueur [...]. Ce monsignor me paraît bien dessalé ; je me forme beaucoup avec lui, et je me sens déjà tout autre (223-224).

Le modèle du genre induit la réflexion critique avec réalisme et sobriété. Voltaire se concentre quant à lui sur des cas particuliers grivois et peu crédibles, jouant de la candeur absolue de ses épistoliers et de leur incapacité supposée à apprendre par l'expérience. La satire naît ainsi d'une exagération ostensible du poncif de la naïveté du voyageur étranger. Notons que ce mécanisme de détournement se reproduit en de nombreux autres passages du conte.

Le système de datation adopté par Les Lettres d'Amabed met également en œuvre une amplification humoristique des dispositifs temporels traditionnels du genre persan. Si la datation est un effet de réel élémentaire du récit par lettres (HAROCHE-BOUZINAC, 1995 : 139), le choix d'une convention différente de celle pratiquée par la civilisation chrétienne occidentale crée un effet dépaysant propre au sous-genre en question. Mais, une fois encore, Voltaire infléchit le procédé canonique. Le système de datation des Lettres Persanes, bien que différent de celui du lecteur, possède une logique interne facilement identifiable et est appliqué de manière constante au fil du récit. La première lettre est ainsi datée du « 15 de la lune de Saphar $1711 »(52)$ et chacune des cent soixante et une missives est porteuse d'une date, jusqu'à la dernière, le « 8 de la lune de Rebiab, 1, $1720 »$ (341). Le défilement chronologique du nombre des années est par ailleurs assimilable au système de référence du lecteur. Le dispositif de Voltaire est quant à lui beaucoup plus en décalage avec le réel, comme l'exemplifient ces deux occurrences :

À Bénarès, le second du mois de la souris, l'an du renouvellement du monde 115652 (179).

À Goa, le cinq du mois du tigre, l'an du renouvellement du monde 115652 (188).

La nature hyperbolique du chiffre des années, destinée à souligner l'insignifiance comparative de la civilisation européenne, induit aussi un décalage humoristique avec la fonction traditionnellement authentifiante du dispositif temporel. De plus, l'absence de systématisme et de régularité dans la datation ne permet pas d'inscrire l'histoire dans une durée concrète : seules six lettres sur trente-quatre sont datées, et seules deux d'entre elles mentionnent simultanément un jour, un mois et une année $(179,188)$. Les quelques datations présentes mélangent progressivement éléments occidentaux et orientaux sans fournir d'informations permettant une transposition claire entre les deux normes. Le dispositif temporel des 
Lettres d'Amabed, à la fois caricatural et irrégulier, opère ainsi un brouillage de l'effet de réel normalement inhérent à la datation des missives.

Pour terminer, la mise en scène de l'éditeur fictif du conte est, elle aussi, caractérisée par une tendance au détournement satirique. Initialement conçu comme un effet de réel permettant de masquer l'existence de l'auteur tout en inscrivant le contenu du récit dans la réalité du lecteur (CALAS, 1996 : 48), le péritexte éditorial dépasse très rapidement cette fonction utilitaire pour devenir une composante essentielle du roman épistolaire. Le jeu avec le lecteur sur l'illusion de réalité assignée aux préfaces est donc constitutif du genre dès ses débuts. La préface des Lettres Persanes met en scène un éditeur qui, assumant une fonction de traducteur, présente les lettres au lecteur.

Les Persans qui écrivent ici étaient logés avec moi ; nous passions notre vie ensemble. Comme ils me regardaient comme un homme d'un autre monde, ils ne me cachaient rien. En effet, des gens transplantés de si loin ne pouvaient plus avoir de secrets. Ils me communiquaient la plupart de leurs lettres ; je les copiai [...]. Je ne fais donc que l'office de traducteur : toute ma peine a été de mettre l'ouvrage à nos mœurs. J'ai soulagé le lecteur du langage asiatique, autant que je l'ai pu, et l'ai sauvé d'une infinité d'expressions sublimes, qui l'auraient ennuyé jusque dans les nues (4748).

Dans Les Lettres d'Amabed, œuvre quant à elle dépourvue de préface, l'utilisation de cette fonction romanesque est prétexte à une sortie polémique. De fait, la première page du texte mentionne en guise de sous-titre que les lettres ont été « Traduites par l'abbé Tamponet» (179). Ce docteur de la Sorbonne qui avait joué un rôle dans l'arrêt contre la publication de l'Encyclopédie en 1760 peut être considéré comme un ennemi philosophique de Voltaire. L'auteur subvertit donc à des fins satiriques le potentiel normalement authentifiant du péritexte épistolaire et crée un télescopage entre l'illusion de réalité du récit et le monde de l'auteur (et de son lecteur). La fonction éditoriale d'annotateur, au moyen de laquelle l'éditeur fictif « vient corriger un point de vue, apporter une information, expliquer une allusion, renvoyer à un autre texte » (CALAS, 1996 : 54-55), suit une logique de détournement similaire.

\section{Voltaire, Les lettres d'Amabed}

Le pape est en grand ce que le dalaï-lama est en petit ; s'il n'est pas immortel comme le lama, il est tout-puissant pendant sa vie, ce qui vaut bien mieux. Si quelquefois on lui résiste, si on le dépose, si on lui donne des soufflets, ou même si on le tue* entre les bras de sa maîtresse, comme il est arrivé quelquefois, ces inconvénients n'attaquent jamais son divin caractère.

*Jean VIII, assassiné à coups de marteau par un mari jaloux.

Jean X, amant de Théodora, étranglé dans son lit.

\section{Montesquieu, Lettres Persanes}

Un homme faisait tous les jours à Dieu cette prière : "Seigneur, je n'entends rien dans les disputes que l'on fait sans cesse à votre sujet : je voudrais vous servir selon votre volonté ; mais chaque homme que je consulte veut que je vous serve à la sienne [...] Il m' arriva, l'autre jour, de manger un lapin dans un caravansérail : trois hommes, qui étaient auprès de là, me firent trembler : ils me soutirent tous trois que je vous avais grièvement offensé ; l'un*, parce que cet animal était immonde ; l'autre**, parce qu'il était étouffé, l'autre enfin*** parce qu'il n'était pas poisson.

*Un Juif. 


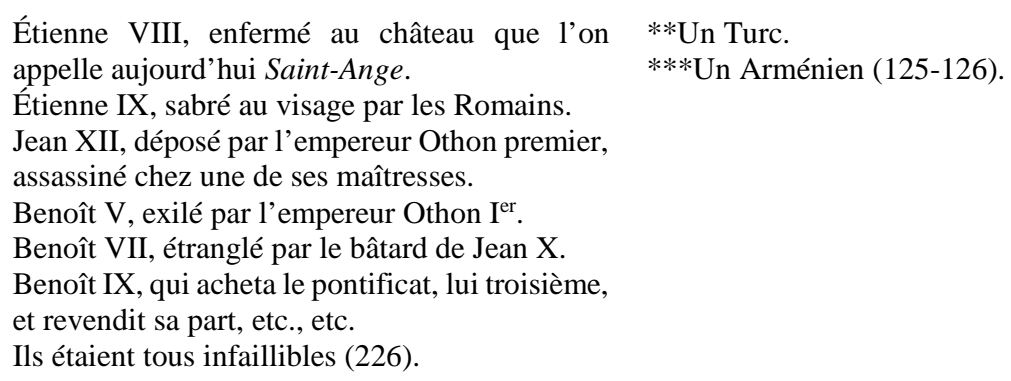

Si les commentaires de l'éditeur fictif de Montesquieu induisent la réflexion critique, la sobriété de leur didactisme - cependant non dénué d'humour - assure la vraisemblance de l'effet de réel romanesque. Voltaire s'inscrit quant à lui une nouvelle fois dans une démarche d'exagération du procédé, utilisant le péritexte éditorial comme vecteur de controverse et de provocation explicite.

\section{L'épistolaire in absentia : silence épistolaire et figuration structurelle de l'acculturation}

\subsection{Une traversée de la Manche : le roman sentimental par lettres}

Les Lettres d'Amabed présentent un second type de jeux d'échos avec le genre épistolaire, liés cette fois à la disparition de certaines composantes plutôt qu'à leur maniement ostensible. C'est plus précisément avec Paméla ; or, Virtue Rewarded de l'anglais Samuel Richardson (1740), archétype de la veine épistolaire sentimentale, que le conte voltairien présente une filiation structurelle. Le roman anglais met en scène les tribulations d'une jeune domestique qui doit protéger sa vertu face aux avances du fils de sa défunte maîtresse. Paméla est ensuite confrontée à la cour effrénée du maître de maison, désormais veuf, et tombé amoureux d'elle après avoir lu son journal. À force de résistance et de vertu, Paméla finit par inspirer l'admiration de ce dernier, qui se repent, l'épouse, et l'introduit dans un nouvel univers social auquel elle doit s'intégrer.

Le succès de l'œuvre est immédiat. Il est toutefois contrebalancé par l'émergence de virulentes critiques taxant d'irréalisme et d'hypocrisie le moralisme chrétien et bourgeois érigé en exemple par le roman. Comme le signale Martial POIRSON dans un récent article (2010: 240-241), celui-ci génère rapidement une véritable "querelle des Paméla », de nombreuses satires paraissant alors en Angleterre. La version française ne se fait pas attendre. Dès 1741 est publiée anonymement une première Paméla ou la Vertu récompensée $e^{3}$, que la critique moderne ${ }^{4}$ attribue périodiquement - et avec précaution - à l'abbé Prévost ou à un

\footnotetext{
${ }^{3}$ Anonyme (1741), Pamela ou la vertu récompensée. Traduit de l'anglais. En deux tomes, Londres, chez Thomas Woodward et Jean Osborn. En raison de l'attribution controversée et de l'histoire éditoriale instable de l'œuvre, les futures références sont à cette première version française identifiée du texte.

${ }^{4}$ Attribution à Aubert de la Chesnaye Des Bois par Hynes (1990:117); attribution à Prévost par HARTMANN (2002: 46) ; mention des deux options par WiLLIAMS (1975: 124); mention de l'existence de deux versions simultanées par PoIRSON (2010 : 238). Cette liste ne reprend pas la totalité des
} 
auteur moins connu, Aubert de la Chesnaye Des Bois. Pierre Hartmann signale à propos de la primo-réception française du texte que «Paméla débarque sur le continent flanquée de ses faux-frères (ou plutôt de ses fausses-sœurs), les antipaméla qui ont proliféré sur le sol anglais » (HARTMANN, 2002 : 46). Du point de vue de la production romanesque, ce sont donc surtout les anti-paméla, plus que l'œuvre-source de Richardson, qui font des émules. Davantage que le roman, c'est le genre dramatique qui offrira au roman anglais les honneurs de l'imitation et de l'adaptation. Ainsi, deux Paméla (l'une par Louis de Boissy, l'autre par Nivelle de la Chaussée) sont représentées à la Comédie-Française dès 1743 (HYNES, 1990 : 117-118), et la fortune scénique de l'œuvre se prolongera par-delà la Révolution.

Bien qu'il méprise le sentimentalisme pratiqué par Richardson, Voltaire se laisse rattraper par le désir de concurrencer le célèbre auteur. Il compose ainsi Nanine, une comédie inspirée de Paméla qui sera jouée sans grand succès à la Comédie Française en 1749 (HYNES, 1990 ; LABRIOLLE et DUCKWORTH, 1994 : $14-$ 28). L'émulation avec Richardson n'est donc pas neuve lorsque Voltaire publie ses Lettres d'Amabed en 1769. Dans une missive du 29 mai de la même année adressée à son ami et confident Nicolas Thieriot, il positionne explicitement le conte épistolaire - dont il ne reconnaît pas ouvertement la paternité - dans une relation de rivalité à la Paméla, indiquant l'existence d'un lien direct entre les deux œuvres.

\begin{abstract}
Avez-vous entendu parler des aventures d'un Indien et d'une Indienne mis à l'inquisition à Goa du temps de Léon dix, et conduits à Rome pour être jugés ? Il y a dans cet ouvrage une comparaison continuelle de la religion et des mours des Brames avec celles de Rome. L'ouvrage m'a paru un peu libre, mais curieux, naïf et intéressant. Il est écrit en forme de Lettres dans le goût de Paméla. Le titre est, Lettres d'Amabed et d'Adaté. Mais dans les six tomes de Paméla il n'y a rien. Ce n'est qu'une petite fille qui ne veut pas coucher avec son maître à moins qu'il ne l'épouse, et les lettres d'Amabed sont le tableau du monde entier depuis les rives du Gange jusqu' au Vatican (D15668).
\end{abstract}

Jonathan Mallinson conclut à partir de cet extrait que «Voltaire reconnaît une ressemblance formelle superficielle avec le roman anglais, mais la similitude s'arrête là » (MALLINSON, 2008 : 227, nous traduisons). Certes, les composantes épistolaires en présence mobilisées par le conte ne présentent pas de similitudes narratives ou thématiques évidentes avec le roman de Richardson. Comme nous l'avons vu, ce sont plutôt les codes d'écriture de la veine épistolaire exotique en général, et la trame narrative du roman de Madame de Graffigny en particulier (MALLINSON, 2008), qui font l'objet d'un détournement actif. Mais, loin d'épargner la veine épistolaire sentimentale, le conte opère, en creux, une réappropriation parodique de l'absence et de l'effacement de l'un des pôles épistolaires du roman de Richardson.

\title{
2.2. Paméla et Amabed : du dialogue épistolaire au journal
}

Pour rappel, les thématiques morales communes aux deux récits sont celles de la vertu persécutée et de l'acculturation : les naifs voyageurs orientaux de Voltaire font l'objet d'injustes persécutions avant de se laisser gagner par les «valeurs »

références écrites quant à l'histoire de Paméla en France, mais uniquement celles utilisées dans le cadre de cette étude. 
occidentales ; tandis que Paméla, modeste domestique, protège longuement sa vertu assiégée avant d'accéder à un monde social nouveau à la faveur de son mariage.

Les Lettres d'Amabed mettent en scène trois personnages-épistoliers : Adaté, son bien-aimé Amabed, et leur père spirituel Shastasid, resté au pays. Comme l'a montré Frédéric CALAS (1996 : 33), la communication épistolaire du conte peut dès lors être schématisée par un triangle de voix. Le nombre le plus important d'échanges se fait entre Amabed et Shastasid, puisque le jeune homme écrit vingtquatre lettres à son aîné, qui n'en enverra que deux en retour. À cette correspondance principale s'ajoutent les sept lettres adressées au même Shastasid par Adaté, qui relaie la plume d'Amabed durant la captivité de ce dernier. Ces missives n'obtiendront qu'une unique réponse. Nulle trace d'échanges au sein du couple, qui voyage conjointement. Les voix épistolaires des deux jeunes gens n'interagissent donc jamais, et sont toujours dirigées vers un même troisième interlocuteur. L'échange épistolaire s'organise ainsi selon deux pôles, dont l'un présente une double face : le premier acteur de l'échange est Shastasid, et le second, comportant quatre mains, est représenté par le jeune couple. C'est cette caractéristique qui motive l'appellation « triangle tronqué » par Frédéric CALAS (1996 : 33). Les trois missives de Shastasid apparaissent dans la première partie du récit, tandis que sa voix se fait silencieuse dès la seconde partie du conte - soit après la libération d'Amabed et le viol d'Adaté par le père Fa tutto. On passe donc d'un échange épistolaire réciproque - bien qu'inégal - à une structure monophonique : il n'y a plus guère qu'Amabed qui prenne désormais la plume, sans obtenir de réponse. La numérotation des lettres envoyées par Amabed à Shastasid reprend d'ailleurs à zéro après l'épisode relatant l'emprisonnement du jeune homme, et le passage au « journal d'Amabed » (206) est explicitement évoqué dès la troisième lettre postcaptivité. L'effacement de Shastasid est en outre figuré par l'évolution des exordes ouvrant les missives du jeune couple : si les lettres de la première partie du conte contiennent une adresse complimentant le destinataire, les mentions directes de son existence se font de plus en plus rares dans le journal d'Amabed (193, 198, 200, 201). Or, le silence du mentor joue un rôle symbolique prépondérant pour la lecture morale du conte. En effet, les interventions initiales de Shastasid représentent clairement la raison, la vertu et la sagesse. Elles constituent un contrepoids à la candeur caricaturale du couple : Shastasid est celui qui jette un regard critique sur le monde occidental et dénonce explicitement son hypocrisie et sa perversion, comme en témoignent les extraits suivants :

Mon cher fils, je crains mortellement l'irruption des barbares d'Europe dans nos heureux climats [...] Je veux croire que ton docteur est un homme de bien ; mais l'Éternel nous permet de nous défier de ces étrangers (182).

Je ne m'étonne pas que des amateurs du vin, tels que les peuples occidentaux, fassent un si grand cas de celui qui, selon eux, plante la vigne ; mais sois sûr que Noé a été ignoré de toute l'Antiquité connue (185).

Quoi ! tendre épouse de mon cher disciple, tu ne vois pas que le père Fa tutto est un scélérat qui t'a fait tomber dans un piège? Tu ne vois pas que c'est lui seul qui a fait enfermer ton mari dans une fosse, et qui t'y a plongée toi-même pour que tu lui eusses l'obligation de t'en avoir tirée ? Que n'exigera-t-il pas de ta reconnaissance (196)? 
Le mutisme subséquent de ce tuteur-interprète figurerait dès lors «l'idéalisation et [...] la disparition de l'instance morale, qui déserte systématiquement le conte voltairien »(CAMBOU, 2003 : 736B). Barbara Toumarkine abonde en ce sens, signalant que «l'unique destinataire des lettres, qui constitue également l'instance morale du roman, se voit donc rapidement évacué. Il s'évanouit en fait à mesure que ses deux disciples se corrompent » (1998: 21). La disparition progressive de l'une des voix de l'échange épistolaire symbolise donc l'acculturation - et la dissolution morale concomitante - des protagonistes.

Ce schéma semble reproduire la symbolique des voix épistolaires propre à Paméla. La première partie de l'œuvre est composée de trente-deux lettres qui se répondent selon une logique initialement dialogique. Trois personnages sont impliqués dans l'échange : la jeune Paméla et ses deux parents. Toutefois, ces derniers répondent toujours conjointement et peuvent donc être assimilés à un pôle épistolaire unique. Comme celle de Shastasid dans le conte de Voltaire, la voix des parents est d'emblée associée à la morale, la vertu et la raison, comme l'illustre cet extrait de la seconde lettre du roman, la première envoyée par les parents de l'héroïne :

Mais ce qui nous inquiète le plus, c'est la crainte où nous sommes que te voyant élevée si fort audessus de ton rang, tu ne te laisses entraîner à commettre quelque chose de honteux et de criminel. Tout le monde dit que tu es devenue grande \& bien faite ; quelques-uns ajoutent que tu es fort jolie, \& en vérité, si tu n'étais pas ma fille, je l'aurais cru aussi lorsque je te vis la dernière fois il y a six mois [...] Si donc tu nous aimes, si tu fais cas de la bénédiction de Dieu, si tu as quelque égard pour ton propre bonheur à venir, nous t'ordonnons l'un \& l'autre d'être sur tes gardes, \& si tu t'aperçois qu'on entreprenne la moindre chose contre ta vertu, ne manque pas de quitter tout, $\&$ de nous venir trouver au plus tôt. Nous aimons mieux te voir couverte de haillons, \& aller même à ton enterrement, que si l'on pouvait dire, qu'une fille qui nous appartient a préféré des avantages temporels à sa vertu (I, 6-7).

Les quatre réponses des parents aux premières alarmes de leur fille se situent en tout début de récit (lettres II, VIII, XIII, et XVII). Dans la dernière de ces missives, les parents encouragent leur fille à fuir les pressions subies dans la demeure de ses maîtres pour les rejoindre, en dépit de leur situation financière difficile (I, 44-45). Cependant, comme le rapporte la lettre suivante, la jeune fille naïve ne peut se résoudre à ce choix radical et repousse sa décision (I, 45-48). Le dialogue épistolaire devient dès lors tronqué : Paméla adressera dix-huit autres lettres à ses parents, mais celles-ci resteront sans réponse. À la suite de la trente-deuxième lettre (I, 146) - qui n'est jamais explicitement clôturée par une formule de politesse ou une signature l'échange épistolaire glisse, ici aussi, vers la forme du journal : la numérotation des missives disparait, de même que les adresses liminaires et les formules de politesse finales. La jeune fille, désormais séquestrée par son maître après s'être décidée à tenter un retour vers la demeure familiale, retranscrit ses mésaventures jour après jour, et parfois heure après heure, jusqu'à la fin du récit. L'amélioration de sa situation suite à son mariage ne marque pas le retour au dialogue épistolaire : la forme du journal subsiste. La disparition de la seconde voix épistolaire figure structurellement la dissolution de l'instance morale originelle et l'éloignement des valeurs tutélaires au fur et à mesure de l'acclimatation à un nouvel environnement 
social. On le voit, Les Lettres d'Amabed, etc. reproduit la symbolique épistolaire de Paméla, à savoir la représentation de l'acculturation progressive des protagonistes par l'effacement du lien épistolaire avec leur milieu - géographique et/ou social d'origine (le père spirituel des héros voltairiens, les parents de l'héroïne de Richardson).

Mais, cette fois encore, Voltaire détourne le dispositif originel : alors que Paméla réalise un transfuge social en préservant sa vertu, les épistoliers voltairiens s'acclimatent à la débauche et au vice avec une inconscience et une légèreté qui frisent l'absurdité. Leur corruption morale définitive est humoristiquement illustrée par leur partie à quatre avec deux prélats catholiques. Évoqué dans la dernière missive du récit, cet épisode clôt éloquemment les aventures européennes des deux Orientaux.

\begin{abstract}
Notre Monsignor nous donna un excellent repas, avec deux jeunes gens vêtus de rouge de la tête aux pieds [...] Ces deux gentilshommes, en dînant avec nous, proposèrent de nous mener passer quelques jours à leur maison de campagne, car c'est à qui nous aura. Après s'être disputé la préférence le plus plaisamment du monde, Faquinetti s'est emparé de la belle Adaté, et j'ai été le partage de Sacripanté, à condition qu'ils changeraient le lendemain, et que le troisième jour nous nous rassemblerions tous quatre. Déra était du voyage. Je ne sais comment te conter ce qui nous est arrivé, je vais pourtant essayer de m'en tirer (233-234).
\end{abstract}

Nous l'avons vu : cette scène campagnarde peut être lue comme une dévalorisation parodique de la fin du roman de Madame de Graffigny, dans lequel le processus d'acculturation de l'héroïne s'accompagnait d'un accomplissement moral lié au choix d'une vie libre (MALLINSON, 2008 : 231). Cette fin constitue toutefois également un détournement du lien entre effacement des voix épistolaires et portée morale de l'acculturation tel qu'il est mis en scène dans Paméla. En effet, malgré la mise en place d'un dispositif phonique dont la symbolique morale est similaire à celle de son homologue britannique, la fin que choisit Voltaire marque un net contraste avec le commentaire final de l'éditeur fictif de Paméla.

Pour l'aimable Paméla ; elle jouit plusieurs années de suite de la récompense due à sa vertu, à sa piété, \& à sa charité : elle fit les délices de tous ceux \& de celles qui la connurent, \& fut regardée comme le modèle le plus accompli de son siècle et de son sexe (II, 458).

Sans surprise, Voltaire n'exploite le modèle anglais que dans le but de subvertir le puritanisme conservateur de son idéologie. L'institution catholique et les mœurs européennes, cibles privilégiées du patriarche, sont égratignées au passage.

\title{
Conclusion
}

Seul conte de Voltaire à adopter une forme épistolaire, Les Lettres d'Amabed n'ont suscité qu'un enthousiasme modéré. Pourtant, le texte possède un intérêt théorique non négligeable dès lors qu'on l'aborde comme un badinage avec les codes de l'écriture épistolaire plutôt que comme une tentative bona fide de composer un roman par lettres original. Les Lettres d'Amabed se présentent en effet comme un prisme parodique qui réfracte les composantes structurelles et génériques de 
plusieurs des œuvres qui jouèrent un rôle majeur dans l'essor du roman épistolaire, genre souvent critiqué par Voltaire.

Le conte entre tout particulièrement en résonance avec trois romans représentatifs des deux paradigmes qui dominaient alors le champ de la fiction épistolaire : les Lettres d'une Péruvienne (Françoise de Graffigny, 1747) et leur modèle les Lettres Persanes (Montesquieu, 1721) pour la veine exotique; et Paméla ou la vertu récompensée (Richardson, 1740) pour la veine sentimentale. Le rapport entre Les Lettres d'Amabed et les Lettres d'une Péruvienne, qui a été étudié ailleurs, est le plus explicite, puisque le premier texte fait figure de réécriture parodique de la trame narrative du second. Les rapports aux deux autres textes sont plus implicites. On a pu voir comment le maniement visible, en présence, de trois composantes épistolaires (description des perceptions du voyageur étranger, datation et localisation exotiques des missives, et mise en scène de l'éditeur fictif du texte) constituait une marque caricaturale de filiation avec le sous-genre persan ; tandis que la récupération de la structure phonique de Paméla (caractérisée par l'absence et la disparition d'un pôle épistolaire), subvertissait implicitement la lecture moraliste du roman de Richardson.

In praesentia comme in absentia, le rapport des Lettres d'Amabed aux grands modèles épistolaires est donc résolument parodique. Si l'ironie mordante et la grivoiserie sont typiques des contes voltairiens, leur manifestation dans l'objet de cette étude est unique en ce qu'elle dépasse ici le contenu thématique de l'œuvre pour viser également ses marqueurs génériques. À la critique sociale et morale s'ajoute donc une métacritique. Plus qu'une satire philosophique, Les Lettres d'Amabed constitue donc aussi - surtout ? - un badinage avec les dispositifs authentifiants qui régissaient le genre épistolaire au XVIII ${ }^{\mathrm{e}}$ siècle. 


\section{BIBLIOGRAPHIE}

Anonyme (1741), Paméla ou la vertu récompensée. Traduit de l'anglais. En deux tomes, Londres, chez Thomas Woodward et Jean Osborn.

CALAS Frédéric (1996), Le roman épistolaire, Paris, Nathan.

CAMBOU Pierre (2003), Lettres d'Amabed, etc. [Les], in : TrousSON Raymond et VERCRUYSSE Jeroom (dir.), Dictionnaire général de Voltaire, Paris, Honoré Champion, p. 736A - 739B.

DoBIE Madeleine (1996), «Langage inconnu » : Montesquieu, Graffigny and the writing of exile, in : Romanic Review, $\mathrm{n}^{\circ} 87$ (2), p. 209-224.

GRAFFIGNY Françoise de (2002), Lettres d'une Péruvienne, MALLINSON Jonathan (éd.), Oxford, Voltaire Foundation, coll. «Vif».

GRASSI Marie-Claire (1998), Lire l'épistolaire, Paris, Dunod.

GRAYSON Vera L. (1996), The genesis and reception of Mme de Graffigny's Lettres d'une Péruvienne and Cénie, in : Studies on Voltaire and the Eighteenth Century, vol. 336, Oxford, Voltaire Foundation, p. 1-152.

HAROCHE-BouZINAC Geneviève (1995), L'épistolaire, Paris, Hachette.

HowELLS Robin (1987), Processing Voltaire's Amabed, in : British Journal for Eighteenth-Century Studies, vol. 10, n², p. 153-162.

Howland John W. (1991), The Letter Form and the French Enlightenment. The epistolary Paradox, Peter Lang, New-York.

HYNES Peter (1990), From Richardson to Voltaire : Nanine and the novelization of comedy, in : The Eighteenth Century, vol. 31, n², p. 117-135.

MALLINSON Jonathan (2008), Les Lettres d'Amabed : rewriting Graffigny's Lettres d'une Péruvienne, in : CRONK Nicholas (éd.), Voltaire and the 1760s. Essays for John Renwick, Studies on Voltaire and the Eighteenth Century, vol. 2008 : 10, Oxford, Voltaire Foundation, p. 227-235.

MERVAUD Christiane (1991), «L'ongle du lion caduc » : les dernières œuvres de Voltaire devant la critique, in : Euvres et Critiques, vol. 16, n², p. 71-84.

MonTESQuiEu (2003), Lettres Persanes, STAROBINSKY Jean (éd.), Paris, Gallimard, Folio Classique.

POIRSON Martial (2010), Paméla en France ou les prospérités du vice, in : BONNECASE Denis et GENTON François (dir.), Ferments d'Ailleurs - Transferts culturels entre Lumières et romantismes, Grenoble, ELLUG, coll. " Hors collection », p. 229-250.

SHOWALTER English (2004), Françoise de Graffigny. Her life and works, in : Studies on Voltaire and the Eighteenth Century, vol. 2004 : 11, Oxford, Voltaire Foundation.

SMITH David (1990), The popularity of Mme de Graffigny's Lettres d'une Péruvienne : The Bibliographical Evidence, in : Eighteenth-Century Fiction, vol. $3, \mathrm{n}^{\circ} 1$, p. 1-20.

TOUMARKINE Barbara (1998), Quelques remarques sur la fin éludée des Lettres d'Amabed de Voltaire, in : Littératures, n³8, p. 19-32.

VERSINI Laurent (1979), Le roman épistolaire, Paris, Presses universitaires de France. 
VOLPILHAC-AUGER Catherine (2004), Montesquieu et Mme de Graffigny : regards croisés, regards obliques, ou Histoire d'un Persan et d'une Péruvienne, in : MALLINSON Jonathan (éd.), Françoise de Graffigny, femme de lettres. Écriture et réception, Studies on Voltaire and the Eighteenth Century, vol. 2004 : 12, Oxford, Voltaire Foundation, p. 159-168.

Voltaire, Les Lettres d'Amabed (1992), in : DelofFRe Frédéric, VAN DeN Heuvel Jacques et HellegouarC'H Jacqueline (éds.), Candide et autres contes, coll. «Folio Classique », Paris, Gallimard.

Voltaire (1994), Nanine, in : LABRIOlle Marie-Rose et DUCKWORTH Colin (éds.), Les Euvres Complètes de Voltaire, vol. 31B, Oxford, Voltaire Foundation, p. 1-180.

\section{Ressources électroniques}

MCNAMEE Robert et al. (éds.) (2011), Electronic Enlightenment Correspondence, University of Oxford. 